\title{
Usual and Unusual Musculoskeletal Sequalae of COVID 19!
}

\author{
Vaibhav Bagaria' ${ }^{1}$ \\ Received: 16 April 2021 / Accepted: 24 April 2021 / Published online: 3 May 2021 \\ (c) Indian Orthopaedics Association 2021
}

\begin{abstract}
Today the orthopedic world with respect to COVID remains focused on resumption of elective surgery and impact of the pandemic on scientific activities. However, it is equally important that we keep our eyes on the phenomenon that is quietly unraveling in front of the eyes. The phenomenon of post-COVID musculoskeletal sequelae may become a major orthopedic public health problem in coming months and needs to be investigated in depth. The usual post-COVID symptoms include arthralgia and myalgia that are common in many viral diseases. It is the unusual symptoms like occurrence of primary septic joint infection and elevated rates of post-operative infections that warrant attention and further investigation. Could this be attributed to immune consumption leading to a phase of temporary immunosuppression is a matter of speculation and hypothesizing. A short communication here reveals a glimpse of musculoskeletal sequalae that COVID may bring in coming months. These could be usual arthralgia or myalgia which are self-limiting but could also be more sinister like spontaneous osteonecrosis and primary joint infections.
\end{abstract}

COVID 19 virus and disease has shown to affect various systems in affected individual. This affliction has been patient specific and for reasons unknown the impact has been nonuniform. There are numerous studies reflecting on the clinical manifestations of COVID 19. Orthopedic manifestations of COVID 19 infection in patients who presented with the disease or in the immediate aftermath of disease, however, is also not well documented.

A total of 6200 patients were seen in the pandemic period (15th March 2020 to 31 March 2021) in the orthopedic OPD and emergency department of a tertiary care hospital. Of these 90 patients who reported (RTPCR positive in last 3 months) or investigated and found (elevated Ig G titers) having recent COVID came for an orthopedic consult for possible post-COVID Sequalae. Following were the clinical manifestations of the disease from the orthopedic standpoint:

1. Arthralgia and Myalgia: 62

2. Synovitis: 14

3. Acute Primary septic joint: 2

4. Spontaneous osteonecrosis: 2

Vaibhav Bagaria

bagariavaibhav@gmail.com

1 Department of Orthopaedic Surgery, Sir H N Reliance Foundation Hospital, Girgaum, Mumbai, Maharashtra 400004, India
5. Soft tissue Abscess: 4

6. Postoperative infection: 6

Arthralgia and myalgias are not very specific and may be a chance occurrence [1]. These maybe considered usual symptoms for any viral disease. However, the high rate of post-operative infections in those operated in post-COVID period, occurrence of primary septic arthritis, spontaneous osteonecrosis and soft tissues abscess is unusual and noteworthy. While no causal relationship between the symptoms and disease could be established, the association of certain rare condition like acute primary septic joints and spontaneous necrosis is a cause of worry and should be a subject of further investigations. It is pertinent to note that not all patient had symptomatic COVID infection or received steroids. In light of these, the cause is hypothetically attributed to phenomenon of 'Consumption Immunocompromisation' in which the COVID infection utilizes the available immune defense mechanism of the body leaving the patient vulnerable to infections. Similarly, a hypercoagulable state may be responsible for spontaneous osteonecrosis.

Today the orthopedic world with respect to COVID understandably remains focused on resumption of elective surgery and impact on scientific activities [2]. However, it is equally important that we keep our eyes on the phenomenon that is unraveling quietly in front of the eyes. The phenomenon of post-COVID musculoskeletal squeal that may 
become a major orthopedic public health problem in coming months needs to be investigated in depth to reach a meaningful conclusion and to design a preemptive strategy.

\section{Declarations}

Conflict of Interest The article has not been submitted anywhere else and the authors do not declare any conflict of interest.

Ethical Standard Statement This article does not contain any studies with human or animal subjects performed by the any of the authors.

Informed Consent For this type of study, informed consent is not required.

\section{References}

1. Memari, A., Shariat, A., \& Anastasio, A. T. (2020). Rising incidence of musculoskeletal discomfort in the wake of the COVID-19 crisis. Work, 66(4), 751-753. https://doi.org/10.3233/ WOR-203221

2. Chhabra, H. S., Bagaria, V., Keny, S., Kalidindi, K. K. V., Mallepally, A., Dhillon, M. S., Malhotra, R., \& Rajasekharan, S. (2020). COVID-19: Current knowledge and best practices for orthopaedic surgeons. Indian J Orthop, 54(4), 1-15. https://doi. org/10.1007/s43465-020-00135-1 